\title{
Industry update: what is new in the field of therapeutic delivery?
}

\author{
Elaine Harris*, 1 \\ ${ }^{1}$ Innovation21, Unit B, Deansgrange Business Park, Blackrock, Co Dublin, Ireland \\ * Author for correspondence: elaine@innovation21.ie
}

The present industry update covers the period 1-30 November 2017. Sources of information include company press releases, regulatory and patent agencies' notices, scientific literature and various news websites. A number of companies reported positive clinical trial results for therapeutic candidates enabled by different delivery strategies including Vascular Therapies, Adapt and Altemia. November also saw the announcement of some significant collaborations and acquisitions; Cerenis Therapeutics acquired Lypro Biosciences, which gives them access to a proprietary drug delivery nanotechnology platform, NanoDisk ${ }^{\circledR}$, and Takeda announced a collaboration with Portal Instruments to develop a needle-free delivery device for its biological therapeutics. From a patenting perspective, Allergan's strategy of transferring the rights of some of their patents to Saint Regis Mohawk Tribe has drawn scrutiny (and criticism) from US Senators.

First draft submitted: 11 December 2017; Accepted for publication: 9 January 2018; Published online: 9 February 2018

Keywords: competitor intelligence $\bullet$ emerging technologies

\section{Business development}

Torque announces the launch of a platform for a new class of Deep-Primed ${ }^{\mathrm{TM}}$ immunotherapeutics On 14 November, the Massachusetts-based immuno-oncology company, Torque, announced that it had launched a proprietary technology platform to create a new class of immune cell therapeutics to treat cancer. The launch was backed by a US\$ 25 -million investment of Series A capital by Flagship Pioneering. Torque's Deep-Priming ${ }^{\text {TM }}$ technology platform is one that relies on a method of creating anchored drug 'backpacks' using a two-step process to polymerize the immune-modulator drugs with copies of themselves to form the 'backpack', which consists of the drug, connected with a reversible cross-linker and then chemically link this 'backpack' to the carrier T cell, anchoring it to proteins on the T-cell surface.

At the launch, the co-founder of Torque, as well as Professor of Materials Science \& Engineering and Biological Engineering at the Massachusetts Institute of Technology and Investigator with the Howard Hughes Medical Institute (MD, USA), Darrell Irvine, PhD, stated: "By arming immune cells to function robustly deep in the tumor microenvironment, this approach has the potential to create a new class of cellular immunotherapeutics, substantially expanding the efficacy of conventional cell-based methods" [1].

\section{Cerenis Therapeutics acquire Lypro Biosciences}

Cerenis Therapeutics (Toulouse, France) announced on 8 November that it had acquired the California-based Lypro Biosciences. No financial details of the deal were disclosed. Cerenis Therapeutics is focusing on the discovery and development of high-density lipoprotein (HDL) based innovative therapies for the treatment of metabolic and cardiovascular diseases, and Lypro has developed proprietary drug delivery nanotechnologies. Lypro's technology is known as 'NanoDisk ${ }^{\circledR}$ ' and is based on self-assembling, targetable, nanoscale bioparticles allowing for the encapsulation of active drugs into the stable and water-soluble NanoDisk. It is anticipated that the combination of Cerenis' HDL and Lypro's NanoDisk technologies will allow for the development of an HDL particle delivery platform focused on immuno-oncology and novel chemotherapeutic delivery technologies. The company intends to carry out initial Phase I studies, evaluating the technology's safety in delivering active drugs to cancer tissues by the end of 2019 [2]. 


\section{Collaboration announced between Takeda \& Portal Instruments}

Japanese company Takeda announced on 7 November that it had concluded a collaborative agreement with Massachusetts-based Portal Instruments. The aim of the agreement is to facilitate the development of new products combining Portal's needle-free drug delivery device with Takeda's biologic medicines, both at investigational or approved stage.

The first of Takeda's actives to benefit from this collaboration will be the monoclonal antibody Entyvio ${ }^{\circledR}$ (vedolizumab). Entyvio is indicated for the treatment of ulcerative colitis Crohn's disease in adults and is currently administered via intravenous infusion.

Portal Instruments' needle-free delivery technology has been licensed from the Massachusetts Institute of Technology. It works by delivering the therapeutic through a pressurized liquid instead of a needle. This needle-free device is designed to allow self-administration by patients at home [3].

\section{Development \& clinical trials}

\section{Vascular Therapies announces Phase III results}

On 9 November, Vascular Therapies (NJ, USA) announced that they had presented preliminary results on the analysis of the first 18 (open-label) patients from its US Phase III study, for their Sirolimus-eluting Collagen Implant at the annual meeting of the American Society of Nephrology. This study aims to investigate the effect of the Sirolimus-eluting Collagen Implant on arteriovenous fistula outcomes.

It is estimated that approximately $80 \%$ of patients with kidney failure begin dialysis using a catheter (US data). Catheter use is, however, often associated with a high incidence of infection, morbidity and mortality. The preferred alternative by the National Kidney Foundation's to the catheter is to use an arteriovenous fistula. The company is currently enroling patients for its Phase III randomized controlled study. The study will be carried out under a special protocol assessment and it is expected that top-line results will be due in 2019 [4].

\section{Adapt report successful usability data}

Adapt Pharma ${ }^{\circledR}$ (Dublin, Ireland) presented data on 2 November at the 41st Association for Medical Education and Research in Substance Abuse Annual National Conference in Washington, DC, USA on the usability of their nasal naloxone spray NARCAN ${ }^{\circledR}$. The data presented indicated that without either instruction or training, a diverse cross-section of the population (both adults and adolescents, in normal and low-literate categories) could successfully complete the critical steps necessary to deploy NARCAN Nasal Spray appropriately in simulated emergency situations.

Currently NARCAN is the only US FDA approved, needle-free formulation of naloxone for the emergency treatment of opioid overdose. One of the authors of the presentation, Melissa Beck (Concentrics Research, IN, USA) stated: "This ready-to-use nasal spray is designed for effective delivery of this potentially life-saving medication to those experiencing an opioid-related overdose while waiting for emergency medical care to arrive" [5].

\section{Positive data in treatment of sickle-cell disease trials}

Florida-based Sancilio Pharmaceuticals Company, Inc, reported on 1 November successful top-line results to their clinical study evaluating the efficacy and safety of Altemia ${ }^{\mathrm{TM}}$, in pediatric sickle-cell patients aged 5-17 years. Sancilio's Altemia ${ }^{\mathrm{TM}}$ is based on a combination of specific lipids formulated using a proprietary Advanced Lipid Technologies ${ }^{\circledR}$ platform. Altemia has been formulated to restore the appropriate balance to blood cell membranes affected by the disease.

The rationale for the approach taken by Sancilio is that currently available treatments for lipid disorders and their related diseases are inconsistently absorbed and bioavailable, and that they are impacted by the presence or absence of food. Sancilio claims that lipid-based active pharmaceutical ingredients (APIs) encapsulated in using their Advanced Lipid Technologies platform, will have increased bioavailability and more consistently absorbed than existing conventional formulations $[6,7]$.

\section{iVeena reports conclusion of Phase I/lla trial employing its sustained release implant}

iVeena, the Utah-based biopharmaceutical company whose focus is innovative ophthalmological products report results on 9 November for a pilot clinical trial for its leading product, IVMED-10. This product employs iVeena's proprietary intracapsular drug delivery platform and is a bioerodible implant for the treatment of pain and 
inflammation after cataract surgery. IVMED-10 is designed to sustainably deliver dexamethasone postoperatively over the course of 2 weeks and it is placed in the lens capsule at the time of cataract surgery.

Discussing the results of the trial iVeena's CEO, Jerry Simmons said: "This study was designed to measure the safety of IVMED-10 and it met the primary objectives. These results support the ongoing clinical development of IVMED-10 and warrant further investigation into the intracapsular platform" [8].

\section{Cerenis announces study for labeled nanoparticles}

Cerenis Therapeutics announced on 22 November that the first patients have been enroled into its TARGET study. The aim of this study is to evaluate HDL nanoparticles in patients with esophageal cancer. It has been designed to study the potential of labeled HDL nanoparticles (CER-001) to visualize tumors in cancer patients and it is hoped to lead to the opportunity to treat cancer patients using HDL nanoparticles as a specific drug delivery platform capable of targeting tumors. The aim of the TARGET study is to assess the concentrations of Zirconium $89\left({ }^{89} \mathrm{Zr}\right.$ )-labeled CER-001 in tumor tissue in patients with esophageal cancer. Following Cerenis's acquisition of the Californian company Lypro earlier this month, the company intends to employ the NanoDisk ${ }^{\circledR}$ technology, an engineered complex of recombinant human apoA-I, designed to specifically target malignant cells, while sparing healthy cells [9].

\section{Theravance Biopharma \& Mylan submit new drug application to FDA for revefenacin}

The 13 November saw the announcement by Theravance Biopharma and Mylan N.V. (Dublin, Ireland) that they have submitted a new drug application (NDA) to the FDA for their long-acting muscarinic antagonist, revenacin (TD-4208). Revenacin is a once-daily, nebulized bronchodilator under investigation for the treatment of chronic obstructive pulmonary disease. The submission follows on from two pivotal Phase III efficacy studies and a 12-month, open-label, active comparator safety study previously carried out by the companies.

A strategic collaboration was formed by Theravance Biopharma and Mylan N.V. (and their respective affiliates) with the aim of developing and commercializing nebulized revefenacin products for chronic obstructive pulmonary disease and other indications. Under the terms of the agreement, it specifies that Theravance Biopharma takes the lead for the US development program for the revefenacin inhalation solution product. Also under this agreement, all costs related to the registrational program will be reimbursed by Mylan up to the approval of the first NDA, after that costs will be shared between the companies. Mylan will be responsible for the development and commercialization outside of the USA. It is thought that Theravance Biopharma will receive up to $\$ 220$ million in the development and sales milestone payments and profit-sharing arrangement with Mylan and in US sales and royalties on sales outside the USA. Theravance Biopharma will also retain worldwide rights to revefenacin employing other dosage formulations and the rights to nebulized revefenacin in China $[10,11]$.

\section{Oramed to initiate clinical study with its oral insulin capsule}

Oramed Pharmaceuticals (Jerusalem, Israel) announced on 14 November that it had received approval from Israel's Ministry of Health to commence a clinical trial of its oral insulin capsule ORMD-0801 in patients with nonalcoholic steatohepatitis. The proposed 3-month plans to assess the effectiveness of ORMD-0801 in reducing liver fat content, inflammation and fibrosis in patients with nonalcoholic steatohepatitis. Permission for this clinical study follows on from preclinical research, which indicated that their proprietary oral insulin capsule has the ability to reduce inflammation of the liver.

Oramed's ORMD-0801 utilizes their patented Protein Oral Delivery $\left(\mathrm{POD}^{\mathrm{TM}}\right)$ - which is an encapsulation process designed to protect delivered proteins from enzymatic degradation within the gastrointestinal tract and to facilitate improved absorption across the intestinal wall by employing absorption enhancer supplements [12].

\section{TherapeuticsMD to resubmit NDA for TX-004HR}

TherapeuticsMD, Inc (FL, USA) announced on 29 November that it had resubmitted to the FDA its NDA for TX-004HR. TX-004HR is a vaginal softgel capsule designed to be delivered vaginally without the need for an applicator for the treatment of moderate-to-severe vaginal pain during sexual intercourse.

In August, the company had announced that the FDA had issued a General Advice Letter stating that following an initial review of their application, additional endometrial safety information to the NDA for TX-004HR was required to be submitted on or before 18 September 2017, including the safety data from a very large, observational study of long-term, real-world users of vaginal estrogens that had pending publication. 
The FDA requested approximately 6 weeks to perform a comprehensive review of these data and has requested a meeting with the company to discuss the outcome of this review. A tentative meeting date of 3 November 2017 has been set by the Agency. At this meeting, the company expects to learn if this additional endometrial safety data addresses the lack of long-term safety identified in the Complete Response Letter (CRL). The company currently plans to resubmit the NDA for TX-004HR shortly thereafter [13].

\section{Orphan drug status received for iVeena's IVMED-80}

iVeena Delivery Systems, the Utah-based ophthalmic-focused biopharmaceutical company announced on 8 November that the FDA had granted orphan drug designation for its investigational product for the treatment of keratoconus, IVMED-80. Keratoconus is a degenerative disorder which can cause cornea to progressively thin and become conical in shape. This can seriously distort vision and may lead to a need for a corneal transplant. iVeena's IVMED-80 is a noninvasive, proprietary eye-drop, which allows for the nonsurgical, nonlaser treatment for medical cross-linking of the cornea. It is anticipated that the company will begin clinical development early in 2018 [14].

\section{First FDA submission of a combination product for Sensile Medical's SensePatch technology}

Sensile Medical (Olten, Switzerland) announced on 6 November that scPharmaceuticals (MA, USA) has submitted a combination product, Furosemide together with Sensile's patch-pump delivery system for subcutaneous administration for approval from the FDA. This is the first regulatory submission in the US for Sensile Medical's SensePatch technology as a combination product.

The NDA for the combination product of a novel furosemide formulation and sc2Wear Infusor ${ }^{\mathrm{TM}}$ based on the SenseCore micropump technology was submitted in August 2017 and has been accepted for filing under the Prescription Drug User Fee Act by the FDA.

Sensile's SenseCore micropump technology has been designed to facilitate slow and controlled subcutaneous drug delivery to offer a therapeutically equivalent alternative to intravenous or intramuscular drug administration [15].

\section{FDA approval for first study on blood-brain barrier disruption using Exbalate Neuro}

Israel-based INSIGHTEC, announced on 27 November that the FDA had approved the first study to evaluate the safety and feasibility of their Exablate Neuro technology for disrupting the blood-brain barrier in patients with glioblastoma. Exablate Neuro is a magnetic resonance guided focused ultrasound technology. It allows for the injection of a sonographic microbubble solution into the bloodstream of a patient with a malignant brain tumor. Ultrasound waves are then delivered to oscillate the microbubbles, causing temporary disruption of the blood-brain barrier. This trial will include up to 15 subjects with suspected glioblastoma who are scheduled to undergo tumor resection.

Exablate Neuro is the first focused ultrasound device approved by the FDA to treat medication refractory essential tremor patients with noninvasive magnetic resonance guided focused ultrasound thalamotomy and it is anticipated that this study will allow the company to expand into the treatment of glioblastoma [16].

\section{First patients enrolled in NanoPac ${ }^{\circledR}$ clinical trial}

On 7 November, the Dallas, TX based company NanOlogy LLC announced the enrolment of the first patient in a clinical trial of $\mathrm{NanoPac}^{\circledR}$ for mucinous cystic neoplasms (MCNs) of the pancreas treatment. The safety and preliminary efficacy will be evaluated in this Phase II dose-rising trial of NanoPac delivered via endoscopic, ultrasound-guided fine needle injection directly into the MCNs. NanoPac is a sterile suspension of nanoparticulate paclitaxel. Currently, there is no approved drug treatment for patients with MCNs who are judged to be at high risk for progression to cancer. NanOlogy's Vice President of Medical Affairs, Gere Dizerega commented: "This clinical trial is the first study in humans to examine whether NanoPac injected intracystically will safely chemically ablate the cyst with a high locally sustained concentration of the drug".

NanOlogy has a broad clinical development program for NanoPac sterile suspension, including trials for the treatment of prostate cancer, ovarian cancer, pancreatic cancer and pancreatic mucinous cysts [17].

\section{Early-stage development}

Genisphere reports safety of 3DNA ${ }^{\circledR}$ Nanocarrier

Genisphere (PA, USA) reported on 15 November positive results from a safety study (in Sprague-Dawley rats) that evaluated dose escalation and repeated dose toxicity of the company's $3 \mathrm{DNA}^{\circledR}$ nanocarrier. According to the press 
release, repeated intravenous administration of the maximum achievable dose of the 3DNA nanocarrier did not result in any adverse clinical findings.

Genispheres's patented 3DNA nanocarrier platform technology consists of a scaffold-based structure made from proprietary, synthetic DNA which is formed into flexible branches. $3 \mathrm{DNA}^{\circledR}$ nanocarriers are cross-linked to form a stable multivalent architecture and can be readily formulated to facilitate the delivery of a wide range of therapeutics from small molecules to biologics, and nucleic acids.

Genisphere's pipeline includes therapeutics to treat indications such as ovarian and pancreatic cancers and glioblastoma [18].

\section{Cingulate demonstrates triphasic release profile for its lead compound}

Cingulate Therapeutics LLC (KS, USA) reported on 15 November a set of in vivo pharmacokinetic data demonstrating consistent triphasic release profile of its lead compound, CTX-1301 at the American Association of Pharmaceutical Sciences meeting in San Diego, California. CTX-1301 is a proprietary formulation of the therapeutic dexmethylphenidate hydrochloride, indicated as a first-line stimulant for the treatment of Attention Deficit-Hyperactivity Disorder and the formulation utilizes a proprietary timed release technology, OralogiK ${ }^{\mathrm{TM}}$, developed by BDD Pharma (Glasgow, UK).

The Chairman and CEO of Cingulate Therapeutics, Shane J Schaffer said, "We are very pleased with this first-inhuman data, which validates the drug delivery platform technology. In the near term, we have submitted two pre-IND packages to the Food and Drug Administration and look forward to the initiation of our Phase 1/2 clinical trials in 2018. We believe this data indicates that CTX-1301 and CTX-1302 have the potential to become true once-daily timed release ADHD medications, enhancing patient outcomes and earning a significant share of the \$12 billion ADHD market" [19].

\section{Regulatory news \& approvals}

EMD Serono's new GONAL-f ${ }^{\circledR}$ RFF Redi-ject ${ }^{\circledR}$ pen approved

Merck's (Darmstadt, Germany) biopharmaceutical business in the USA and Canada, EMD Serono (MA, USA), announced on 13 November that it had received approval from the FDA for a redesigned version of its GONAL$\mathrm{f}^{\circledR} \mathrm{RFF}^{*}$ Redi-ject ${ }^{\circledR}$ (follitropin alfa injection) pen injector. The redesigned Redi-ject pen is the second version developed since its initial approval in 2013 and it has been approved for the induction of ovulation and pregnancy in oligo-anovulatory women. Approval has been given if the cause of infertility is functional and not due to either primary ovarian failure or the development of multiple follicles in ovulatory women as part of an Assisted Reproductive Technology cycle [20].

\section{ViiV's Juluca approved as a combination HIV product}

ViiV Healthcare, the London-based global specialist HIV company announced on 21 November that the FDA had approved its combination HIV product Juluca ${ }^{\circledR}$. ViiV Healthcare's majority shareholder is GlaxoSmithKline; Pfizer Inc. and Shionogi Limited are also shareholders. Juluca is the first 2-drug regimen comprising of dolutegravir (ViiV Healthcare), an integrase strand transfer inhibitor, and Janssen Therapeutic's rilpivirine, a non-nucleoside reverse transcriptase inhibitor. The new combination therapeutic is indicated as a maintenance regimen for HIV-1 infected adults who are virologically suppressed (HIV-1 RNA $<50$ copies per $\mathrm{ml}$ ) and who have been on a stable antiretroviral regimen for a minimum of 6 months.

Commenting on the approval John C Pottage, Jr, MD, Chief Scientific and Medical Officer of ViiV said, "Based on the fundamental principle that no one should have to take more medicines than necessary, ViiV Healthcare has put in place a comprehensive 2-drug regimen research and development programme built around the characteristics of dolutegravir. Juluca, our new 2-drug regimen, once-daily, single pill, now provides people living with HIV who are virologically suppressed, the option to reduce the number of antiretrovirals they take, while maintaining the efficacy of a traditional three-drug regimen" [21].

\section{Zubsolv ${ }^{\circledR}$ approval by European Medicines Agency for opioid dependence}

On 20 November, Orexo AB (Uppsala, Sweden) announced that the European Medicines Agency had granted a Marketing Authorization for its novel rapidly disintegrating product, Zubsolv ${ }^{\circledR}$ (buprenorphine and naloxone), for the treatment of opioid dependence.

Zubsolv is a rapidly dissolving sublingual tablet licensed for people with opioid dependence. It is the first such therapy to be approved in Europe with a choice of six different strengths. It is estimated (2016 figures) that there 
are approximately 1.3 million high-risk opioid users in Europe and the advantage of this product is that it offers the potential for finer titration and individualized dosing with fewer tablets compared with existing opioid-dependent treatment options.

President and CEO at Orexo AB, Nikolaj Sørensen, said, "Our ambition is to make Zubsolv available to patients outside the US, and this approval is an important step in realizing this goal. We look forward to continuing our journey with Mundipharma, our partner, reaching out with this unique product globally and introducing an important new option in the care of those suffering from opioid dependence" [22].

\section{Patents}

\section{Allergan: novel patenting strategy}

Allergan's novel patenting strategy whereby it has transferred its patents for Restasis, a dry-eye drug, to the Saint Regis Mohawk Tribe in September has come under scrutiny from the US Senate. The deal resulted in the tribe receiving a payment of $\$ 14$ million when it acquired the patents, and as part of the agreement the tribe will then relicense the patents to Allergan for an annual fee of $\$ 15$ million. From Allergan's perspective, the aim of this move is to ward off challenges to the patents by rival pharmaceutical companies. Under law Native American tribes enjoy immunity as "entities granted sovereign status". It remains to be seen if this strategy will prove to be successful for the company [23].

\section{Financial \& competing interests disclosure}

The author has no relevant affiliations or financial involvement with any organization or entity with a financial interest in or financial conflict with the subject matter or materials discussed in the manuscript. This includes employment, consultancies, honoraria, stock ownership or options, expert testimony, grants or patents received or pending, or royalties.

No writing assistance was utilized in the production of this manuscript.

\section{References}

1. Torque, a Flagship Pioneering Company, Launches Platform to Develop a New Class of Deep-Primed ${ }^{\mathrm{TM}}$ Immune Cell Therapies, Financed with $\$ 25 \mathrm{M}$ Series A and Led by a Veteran Management Team. www.torquetx.com/news/press-releases/torque-launch

2. CERENIS Therapeutics Acquires LYPRO Biosciences Expanding Its HDL Strategy Into Immuno-Oncology and Chemotherapeutic Drug Delivery. www.cerenis.com/images/pdfs/images/pdfs/PR_cerenis_LYPRO_ENG_VF.pdf

3. Takeda and Portal Instruments Announce Collaboration to Develop Needle-Free Drug Delivery Device. www.businesswire.com/news/home/20171107005330/en/Takeda-Portal-Instruments-Announce-Collaboration-Develop-Needle-Free

4. Vascular Therapies Announces Preliminary Results from its Phase 3 AV Fistula Study www.vasculartx.com/news

5. Presents Human Factors Study Data on Usability of NARCAN ${ }^{\circledR}$ (naloxone HCl) Nasal Spray at the 41st Association for Medical Education \& Research in Substance Abuse Annual National Conference. www.prnewswire.com/news-releases/adapt-pharma-presents-human-factors-study-data-on-usability-of-narcan-naloxone-hcl-nasalspray-at-the-41st-association-for-medical-education--research-in-substance-abuse-annual-national-conference-300549044.html

6. Altemia ${ }^{\mathrm{TM}}$, Achieves Successful Clinical Results in Pediatric Patients with Sickle Cell Disease (SCD). www.sancilio.com/press-releases

7. Altemia ${ }^{\text {TM }}$, Achieves Successful Clinical Results in Pediatric Patients with Sickle Cell Disease (SCD). www.businesswire.com/news/home/20171101005538/en/AltemiaTM-Achieves-Successful-Clinical-Results-Pediatric-Patients

8. iVeena Delivery Systems Completes Phase 1/2a Pilot Clinical Study of IVMED-10 for Post-Cataract Inflammation. www.businesswire.com/news/home/20171108005244/en/iVeena-Delivery-Systems-Completes-Phase-12a-Pilot

9. First Patients Enter TARGET Study, Recently Initiated by Cerenis Therapeutics to Evaluate HDL Nanoparticles in Patients with Esophageal Cancer. www.businesswire.com/news/home/20171121006117/en/Patients-Enter-TARGET-Study-Initiated-Cerenis-Therapeutics

10. Theravance Biopharma and Mylan Submit New Drug Application to FDA for Revefenacin (TD-4208) in Adults with Chronic Obstructive Pulmonary Disease. www.prnewswire.com/news-releases/theravance-biopharma-and-mylan-submit-new-drug-applicationto-fda-for-revefenacin-td-4208-in-adults-with-chronic-obstructive-pulmonary-disease-300554562.html

11. Theravance Biopharma and Mylan Submit New Drug Application to FDA for Revefenacin (TD-4208) in Adults with Chronic Obstructive Pulmonary Disease.

http://investor.theravance.com/news-releases/news-release-details/theravance-biopharma-and-mylan-submit-new-drug-application-fda

12. Oramed Receives Regulatory Approval to Conduct Clinical Study for Treatment of NASH with its Oral Insulin Capsule. www.prnewswire.com/news-releases/oramed-receives-regulatory-approval-to-conduct-clinical-study-for-treatment-of-nash-with-itsoral-insulin-capsule-300555401.html 
13. TherapeuticsMD Announces Resubmission of New Drug Application for TX-004HR. https://ir.therapeuticsmd.com/news-releases/news-release-details/therapeuticsmd-announces-resubmission-new-drug-application-tx

14. iVeena Delivery Systems Receives FDA Orphan Drug Designation for IVMED-80 for the Treatment of Keratoconus. www.businesswire.com/news/home/20171108005303/en/iVeena-Delivery-Systems-Receives-FDA-Orphan-Drug

15. First US FDA Submission of Wearable Drug-Device Combination Product Based on Sensile Medical's SensePatch Drug Delivery Technology. www.sensile-medical.com/assets/first-us-fda-submission-of-wearable-drug-device_1.pdf

16. FDA Approves First Study of INSIGHTEC's Exablate Neuro to Open the Blood-Brain Barrier in Glioblastoma Patients. www.insightec.com/news-events/press-releases/2017/fda-approves-first-study-of-insightec-s-exablate-neuro-to-open-the-blood-brainbarrier-in-glioblastoma-patients

17. NanOlogy ${ }^{\mathrm{TM}}$ Announces First Patient Enrolled in a Phase 2 Clinical Trial of NanoPac ${ }^{\circledR}$ for Treatment of Mucinous Cysts of the Pancreas. www.businesswire.com/news/home/20171107005060/en/NanOlogy\%E2\%84\%A2-Announces-Patient-Enrolled-Phase-2-Clinical

18. Genisphere Reports Safety of $3 \mathrm{DNA}{ }^{\circledR}$ Nanocarrier. https://genisphere.com/news/genisphere-reports-safety-3dna\%C2\%AE-nanocarrier

19. Cingulate Therapeutics to Present In Vivo Pharmacokinetic Data Demonstrating Consistent Tri-Phasic Release Profile of Lead Compound at the American Association of Pharmaceutical Scientists (AAPS) Annual Meeting. www.prnewswire.com/news-releases/ cingulate-therapeutics-to-present-in-vivo-pharmacokinetic-data-demonstrating-consistent-tri-phasic-release-profile-of-leadcompound-at-the-american-association-of-pharmaceutical-scientists-aaps-annual-meeting-300556422.html

20. EMD Serono Receives FDA Approval for New GONAL-f ${ }^{\circledR}$ RFF Redi-ject ${ }^{\circledR}$ Pen. www.prnewswire.com/news-releases/emd-serono-receives-fda-approval-for-new-gonal-f-rff-redi-ject-pen-300553801.html

21. ViiV Healthcare announces US FDA approval for Juluca ${ }^{\circledR}$. www.viivhealthcare.com/media/press-releases/2017/november/viiv-healthcare-announces-us-fda-approval-for-juluca.aspx

22. Zubsolv ${ }^{\circledR}$ Receives Authorization for Treatment of Opioid Dependence in Europe. www.prnewswire.com/news-releases/zubsolv-receives-authorization-for-treatment-of-opioid-dependence-in-europe-300559328.html

23. Allergan's unusual legal tactic attracts political scrutiny. www.economist.com/news/business/21731418-how-native-american-tribescan-perhaps-help-protect-patents-allergans-unusual-legal-tactic 
